\title{
Cooperative translational control of polymorphic BAFF by NF90 and miR-15a
}

\author{
M. Laura Idda ${ }^{1, *}$, Valeria Lodde ${ }^{2,3}$, Waverly G. McClusky ${ }^{1}$, Jennifer L. Martindale ${ }^{1}$, \\ Xiaoling Yang ${ }^{1}$, Rachel Munk ${ }^{1}$, Maristella Steri ${ }^{2}$, Valeria Orrù ${ }^{2}$, Antonella Mulas ${ }^{2}$, \\ Francesco Cucca $^{2,3}$, Kotb Abdelmohsen ${ }^{1}$ and Myriam Gorospe ${ }^{\oplus 1, *}$
}

\begin{abstract}
${ }^{1}$ Laboratory of Genetics and Genomics, National Institute on Aging Intramural Research Program, National Institutes of Health, Baltimore, MD 21224, USA, ${ }^{2}$ Istituto di Ricerca Genetica e Biomedica, Consiglio Nazionale delle Ricerche (CNR), 09042 Monserrato, Cagliari, Italy and ${ }^{3}$ Department of Biomedical Sciences, University of Sassari, Sassari, Italy
\end{abstract}

Received June 19, 2018; Revised August 31, 2018; Editorial Decision September 12, 2018; Accepted September 13, 2018

\begin{abstract}
Polymorphisms in untranslated regions (UTRs) of disease-associated mRNAs can alter protein production. We recently identified a genetic variant in the $3^{\prime}$ UTR of the TNFSF13B gene, encoding the cytokine BAFF (B-cell-activating factor), that generates an alternative polyadenylation site yielding a shorter, more actively translated variant, BAFF-var mRNA. Accordingly, individuals bearing the TNFSF13B variant had higher circulating BAFF and elevated risk of developing autoimmune diseases. Here, we investigated the molecular mechanisms controlling the enhanced translation of BAFF-var mRNA. We identified nuclear factor 90 (NF90, also known as ILF3) as an RNA-binding protein that bound preferentially the wild-type (BAFF-WT mRNA) but not BAFF-var mRNA in human monocytic leukemia THP1 cells. NF90 selectively suppressed BAFF translation by recruiting miR-15a to the $3^{\prime}$ UTR of BAFFWT mRNA. Our results uncover a paradigm whereby an autoimmunity-causing BAFF polymorphism prevents NF90-mediated recruitment of microRNAs to suppress BAFF translation, raising the levels of disease-associated BAFF.
\end{abstract}

\section{INTRODUCTION}

Cellular responses to internal and external signals are potently controlled by post-transcriptional gene regulatory processes, including pre-mRNA splicing and mRNA maturation, transport, stability, and translation (1). Accordingly, all cellular events, including proliferation, apoptosis, senescence, differentiation, and the cellular response to im- mune stimuli, are strongly regulated by post-transcriptional factors. Among the most prominent post-transcriptional regulators are RNA-binding proteins (RBPs) and microRNAs; their influence is often elicited through specific sites in 3 '-untranslated regions (UTRs) through which RBPs and microRNAs bind mRNAs and modulate mRNA turnover and/or translation (2-4). Immune responses, both physiologic and disease-associated, are strongly regulated by RBPs and microRNAs $(5,6)$.

The large and heterogeneous family of autoimmune diseases including type-I diabetes, rheumatoid arthritis (RA), systemic lupus erythematosus (SLE), and multiple sclerosis (MS), is characterized by abnormal immune responses to normal body antigens mediated by specific immune cells and their products, leading to inflammation and tissue injury. Over the last decade, genome-wide association studies (GWAS) have uncovered several strong associations between genetic variants and risk of autoimmune disease (7). In a recent GWAS analysis of the Sardinian population, we uncovered the association of MS and SLE with a genetic variant in the $3^{\prime} \mathrm{UTR}$ of the TNFSF13B gene, which encodes the cytokine B-cell-activating-factor (BAFF), a member of the tumor necrosis factor superfamily (8). The causal variant was found to be an insertion-deletion (GCTGT > A, [GCTG/-] rs200748895 and [T/A] rs374039502 in the 1000 Genomes Project-variants data set) where 'A', the minor, risk-associated variant allele that is found with a frequency of 0.265 in Sardinians ('BAFF-var'), created an upstream alternative polyadenylation site (APA). The resulting transcript, $B A F F$-var mRNA, was more actively translated than the long (wild-type) $B A F F-W T$ mRNA, and led to higher production of soluble (s)BAFF. The short variant was more actively expressed partly because it lacked a site of repression by microRNA miR-15a; higher sBAFF in turn increased circulating B cells and immunoglobulins,

\footnotetext{
${ }^{*}$ To whom correspondence should be addressed. Tel: +1 410558 8443; Email: gorospem@grc.nia.nih.gov Correspondence may also be addressed to M. Laura Idda. Email: marialaura.idda@irgb.cnr.it
} 
raising the risk of MS and SLE (8). BAFF is mainly produced by monocytes, macrophages, neutrophils and dendritic cells (9); it binds to B-cell receptors and promotes B cell proliferation and differentiation (10-12). BAFF overexpression resulted in abnormal $\mathrm{B}$ cell-mediated autoimmune responses resulting in an increased risk of MS and SLE (8). Interestingly, the observed differences in SBAFF production and downstream phenotypes were only partially due to changes in the levels of $B A F F$-var mRNA compared with $B A F F$ - $W T$ mRNA (8), suggesting that both mRNA levels and translation rate were regulated.

Here, we investigated the molecular factors that regulate BAFF translation selectively in a polymorphismdependent manner. Using different approaches to identify $B A F F$ mRNA-associated molecules and their impact on BAFF production, we found that the RBP NF90 (ILF3) potently repressed BAFF translation from $B A F F-W T$ mRNA but not from BAFF-var mRNA in THP-1 cells, a human monocytic leukemia line. Further results revealed that NF90 suppressed BAFF production by promoting the interaction of microRNA miR-15a with BAFF-WT mRNA. Our findings suggest that NF90 represses BAFF translation by recruiting miR-15a to $B A F F-W T$ mRNA, and that the polymorphic variant $B A F F$-var, having lost the NF90 binding site, is now constitutively translated, producing higher levels of BAFF known to cause abnormal immune responses and promote disease.

\section{MATERIALS AND METHODS}

\section{Cell culture and transfections}

THP-1 human acute monocytic leukemia cells were cultured in RPMI-1640 medium and human cervical carcinoma HeLa cells were cultured in Dulbecco's modified essential medium, each supplemented with $10 \%$ fetal bovine serum and antibiotics. EBV-transformed B-lymphoblastoid cell lines from genotyped healthy human donors from Sardinia were chosen so as to include both BAFF-WT and BAFF-var alleles. Lymphoblastoid cell lines (LCL) were cultured in RPMI-1640 medium supplemented with $14 \%$ heat-inactivated fetal calf serum, antibiotics, $1 \%$ sodium pyruvate and $1 \%$ L-glutamine.

Control small interfering RNA (Ctrl siRNA) was from Qiagen, and the siRNAs directed to NF90, AGO1, AGO2, AGO3 and AGO4 were from Santa Cruz Biotechnology; miRNA mimics and inhibitors were purchased from Thermo Fisher Scientific and transfected at a concentration of $50 \mathrm{nM}$. Plasmids and small RNAs were transfected using Lipofectamine-2000 (Invitrogen) or electroporation using Amaxa (Lonza).

To generate THP-1 cells expressing constitutively lower levels of NF90, ready-to-use lentiviral particles expressing NF90 shRNA (containing three target-specific constructs that encode 19- to 25-nt short hairpin RNAs (shRNAs) designed to knock down NF90) or scramble control shRNA were purchased from Santa Cruz Biotechnology. Particles were added to THP-1 cells in the presence of polybrene (8 $\mu \mathrm{g} / \mathrm{ml})$. Puromycin $(0.5 \mu \mathrm{g} / \mathrm{ml})$ was added to the culture medium 1 week later to select for cells stably expressing integrated vectors. Pooled NF90-silenced populations were used for experiments.

\section{EGFP-BAFF 3'UTR reporter constructs and Amaxa trans-} fection

The pEGFP-C1 vector, expressing the EGFP coding region, was engineered to express the full-length (WT) $3^{\prime}$ UTR of $B A F F$ mRNA (pEGFP-C1-BAFF-WT $3^{\prime} \mathrm{UTR}$ ) or the shorter variant (var) $3^{\prime} \mathrm{UTR}$ of $B A F F$ mRNA (pEGFPC1-BAFF-var 3'UTR). Plasmid pEGFP-C1-BAFF-WT $3^{\prime}$ UTR was further modified by introducing a deletion of the NF90 site (pEGFP-C1-BAFF 3'UTR( $\triangle$ NF90), eliminating both miR-15a sites [pEGFP-C1-BAFF-WT $3^{\prime}$ UTR (miR-15a mut)], and creating a mutant in which both miR15a sites were moved [pEGFP-C1-BAFF-WT 3'UTR (new miR-15a)]. A naturally occurring stop codon located $50 \mathrm{nt}$ after the beginning of the $3^{\prime}$ UTR was maintained in all the EGFP expression vectors. Constructs were verified by sequencing. Reporter assays were carried out in THP-1 cells on 12-well plates. Cells expressing normal (Ctrl shRNA) or reduced levels (NF90 shRNA) of NF90 were transfected with $500 \mathrm{ng}$ of either reporter construct, or empty vector using Amaxa (Lonza). Twenty-four hours after transfection, EGFP expression was measured by western blot analysis and the relative protein levels quantified by densitometry and normalized to the levels of a housekeeping protein (e.g. HSP90, ACTB).

\section{RNA isolation and RT-qPCR analysis}

RNA was isolated from cells using the TriPure isolation reagent (Roche) and the PureLink RNA mini Kit (Life Technologies) following the manufacturers' protocols. Total RNA was reverse-transcribed into cDNA using Maxima reverse transcriptase (Thermo Fisher) and random hexamers and analyzed by quantitative (q)PCR analysis using SYBR Green mix (Kapa Biosystems) and gene-specific primers listed in Supplementary Table S2. The relative mRNA expression levels were calculated by the $2-\Delta \mathrm{Ct}$ method. miRNAs were reverse-transcribed using MicroRNA first-strand synthesis (Clontech), and quantified by qPCR analysis using $U 6$ as internal control and the primers listed in Supplementary Table S2.

\section{Biotin pulldown and mass spectrometry (MS) analyses}

To synthesize biotinylated RNAs corresponding to the $B A F F$ 3'UTR and control RNAs, PCR fragments were prepared using forward primers that contained the $\mathrm{T} 7$ RNA polymerase promoter sequence [(T7), CCAAGC TTCTAATACGACTCACTATAGGGAGA] and reverse primers listed in Supplementary Table S2. After purifying the DNA template, biotinylated transcripts were synthesized using MaxiScript T7 kit (Ambion); whole-cell lysates $(200 \mu \mathrm{g})$ from THP-1 cells were incubated with $1 \mu \mathrm{g}$ of biotinylated RNA for $30 \mathrm{~min}$ at room temperature (13). Complexes were isolated with Streptavidin-coupled Dynabeads (Invitrogen) and proteins in the pulldown material were analyzed using western blot analysis and MS.

For MS analysis, peptide mixtures from each sample were loaded onto a peptide trap cartridge and eluted onto a reversed-phase PicoFrit column (New Objective, Woburn, MA, USA). Eluted peptides were ionized and sprayed into 
the mass spectrometer, using a Nanospray Flex Ion Source ES071 (Thermo Scientific). The LC/MS/MS analysis of samples were carried out using a Thermo Scientific QExactive hybrid Quadrupole-Orbitrap Mass Spectrometer and a Thermo Dionex UltiMate 3000 RSLCnano System. Proteins were identified using the Thermo Proteome Discoverer 1.4.1 platform. Database search against public human protein database from NCBI was performed through the Proteome Discoverer 1.4.1 platform.

\section{Immunoprecipitation of ribonucleoprotein (RNP) complexes (RIP analysis)}

For RIP analysis, lysates of THP-1 cells were prepared in PEB buffer $\left(10 \mathrm{mM}\right.$ Hepes, $100 \mathrm{mM} \mathrm{KCl,} 5 \mathrm{mM} \mathrm{MgCl}_{2}$, $25 \mathrm{mM}$ EDTA, 0.5\% IGEPAL, $2 \mathrm{mM}$ DTT, $50 \mathrm{U} / \mathrm{ml}$ RNase out and protease inhibitors); after clarification, lysates were incubated $\left(2 \mathrm{~h}, 4^{\circ} \mathrm{C}\right)$ with a suspension of protein-A Sepharose beads precoated with $5 \mu \mathrm{g}$ of antiNF90 or mouse IgG (BD Transduction Laboratories). Following incubation, the beads were washed with NT2 buffer (50 mM Tris- $\mathrm{HCl}$ pH 7.5, $150 \mathrm{mM} \mathrm{NaCl}, 1 \mathrm{mM} \mathrm{MgCl}$, $0.05 \%$ IGEPAL) and subsequently treated with RNase-free DNase I ( $\left.15 \mathrm{~min}, 30^{\circ} \mathrm{C}\right)$, and washed with NT2 buffer. RNA enrichments were analyzed by RT-qPCR using the primers listed in Supplementary Table S2.

\section{Co-immunoprecipitation (IP) analysis}

For co-IP analysis, THP-1 lysates were prepared in PEB buffer followed by 2 freeze/thaw cycles. After clarification and preclearing, lysates were incubated for $16 \mathrm{~h}$ at $4^{\circ} \mathrm{C}$ with protein-A Sepharose beads precoated with $5 \mu \mathrm{g}$ of antiNF90, anti-AGO2 or IgG (BD Transduction Labs). After incubation, the beads were washed in NT2 buffer for $5 \mathrm{~min}$, with rotation. Proteins were detected by western blot analysis.

\section{Western blot analysis and ELISA}

Whole-cell lysates were prepared in RIPA buffer, and proteins were separated using electrophoresis in SDScontaining polyacrylamide gels (SDS-PAGE). Following transfer, nitrile membranes were incubated for $16 \mathrm{~h}$ with primary antibodies recognizing NF90 (BD Transduction Laboratories), BAFF, AGO3, AGO4 (Millipore), AGO1, AGO2 (Abcam), HSP90 $\alpha / \beta$, GAPDH, HSP70, or EGFP (Santa Cruz Biotechnology). Secondary antibodies conjugated with horseradish peroxidase (HRP) (Kindle Biosciences) were detected by enhanced chemiluminescence (Kevik). The levels of soluble BAFF were evaluated using an ELISA Kit (Adipogene).

\section{Polysome gradients}

For polysome analysis, THP-1 or HeLa cells were incubated with $0.1 \mathrm{mg} / \mathrm{ml}$ cycloheximide for $10 \mathrm{~min}$, and cytoplasmic extracts were fractionated through a linear sucrose gradient $[10-50 \%(\mathrm{w} / \mathrm{v})]$ as previously reported (14). Fractions were collected using a fraction collector and monitored by optical density measurement $\left(A_{254}\right)$ (Brandel). The RNA in each fraction was isolated and the relative enrichment was quantified using RT-qPCR analysis and the primers listed in Supplementary Table S2. The polysome data were displayed using the conventional representation of mRNAs on polysome gradients. The analysis was performed multiple times, preparing fresh gradients each time, and collecting RNA from each fraction for RT-qPCR quantification. The $\%$ mRNA in each fraction was then plotted relative to the total levels of that mRNA in the entire gradient. Given the variability in relative distribution among experimental repeats, mainly due to the fact that the gradients are not always identical, representative experiments are displayed in the main text; independent experiments are shown in Supplementary Figure S4B and C.

\section{Biotinylated miRNA pulldown}

THP-1 cells were transfected with biotinylated miR-15a or Ctrl (60 nM each) using Lipofectamine 2000. Forty-eight $\mathrm{h}$ later, cells were washed with PBS twice and lysed in 1 $\mathrm{ml}$ lysis buffer [20 mM Tris ( $\mathrm{pH} 7.5), 100 \mathrm{mM} \mathrm{KCl,} 5 \mathrm{mM}$ $\mathrm{MgCl}_{2}, 0.3 \% \mathrm{NP}-40,100 \mathrm{U}$ of RNase OUT (Invitrogen), protease inhibitor (Roche)], and incubated on ice for 10 min. After clearing the cytoplasmic lysate by centrifugation at $12000 \times \mathrm{g}(10 \mathrm{~min})$, steptavidin-coated Dynabeads (100 $\mu \mathrm{l})$ were added and further incubated $\left(4 \mathrm{~h}, 4^{\circ} \mathrm{C}\right.$ with rotation). Beads were washed four times with $1 \mathrm{ml}$ ice-cold lysis buffer, RNA was isolated using TriPure isolation solution, and the enrichment of $B A F F$ mRNA was measured by RT-qPCR analysis and normalized to the levels of $A C T B$ mRNA or $18 S$ rRNA.

\section{Statistical analysis}

Data are presented as the means \pm standard error of the means (SEM). Significance was tested using two-tailed Student's $t$ test. $P<0.05$ was considered significant.

\section{RESULTS}

\section{$B A F F$-var mRNA is more actively translated}

By GWAS analysis, we recently reported an association between the TNFSF13B gene, encoding the cytokine BAFF, with autoimmune diseases MS and SLE (8). Depicted in Figure $1 \mathrm{~A}$ is the identified variant, an insertion-deletion in the $3^{\prime}$ UTR, GCTGT $>A$, where the minor variant ' $A$ ', the risk allele, created an upstream alternative polyadenylation (APA) signal that gave rise to a shorter transcript, $B A F F$ var mRNA. Using serum from Sardinian participants, we found that individuals with both $B A F F-W T$ alleles $(n=$ 1280) expressed far lower sBAFF levels $(726.7 \mathrm{pg} / \mathrm{ml})$ than individuals with both $B A F F$-var alleles $(n=272,1000.7$ $\mathrm{pg} / \mathrm{ml}$ ) with an associated $P$-value of $8.89 \mathrm{e}^{-82}$ (Figure 1A). Interestingly, these differences were only partially due to changes in the levels of $B A F F$-var mRNA compared with $B A F F-W T$ mRNA (8), suggesting regulation at both levels, increased mRNA levels and increased translation.

To investigate the mechanism through which higher levels of BAFF were produced from $B A F F$-var mRNA, we prepared reporter constructs that expressed chimeric $\mathrm{mR}$ NAs spanning the EGFP (enhanced green fluorescence protein) coding region (black) and the $3^{\prime} \mathrm{UTR}$ from either the 
A
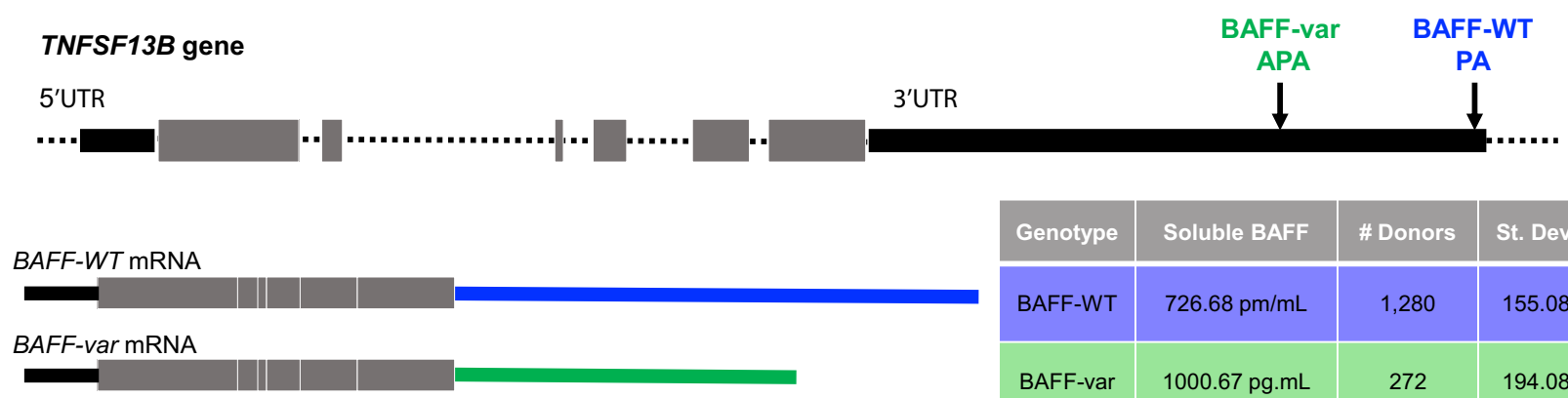

\begin{tabular}{|c|c|c|c|}
\hline Genotype & Soluble BAFF & \# Donors & St. Dev. \\
\hline BAFF-WT & $726.68 \mathrm{pm} / \mathrm{mL}$ & 1,280 & 155.08 \\
\hline BAFF-var & $1000.67 \mathrm{pg} \cdot \mathrm{mL}$ & 272 & 194.08 \\
\hline
\end{tabular}

\section{B}
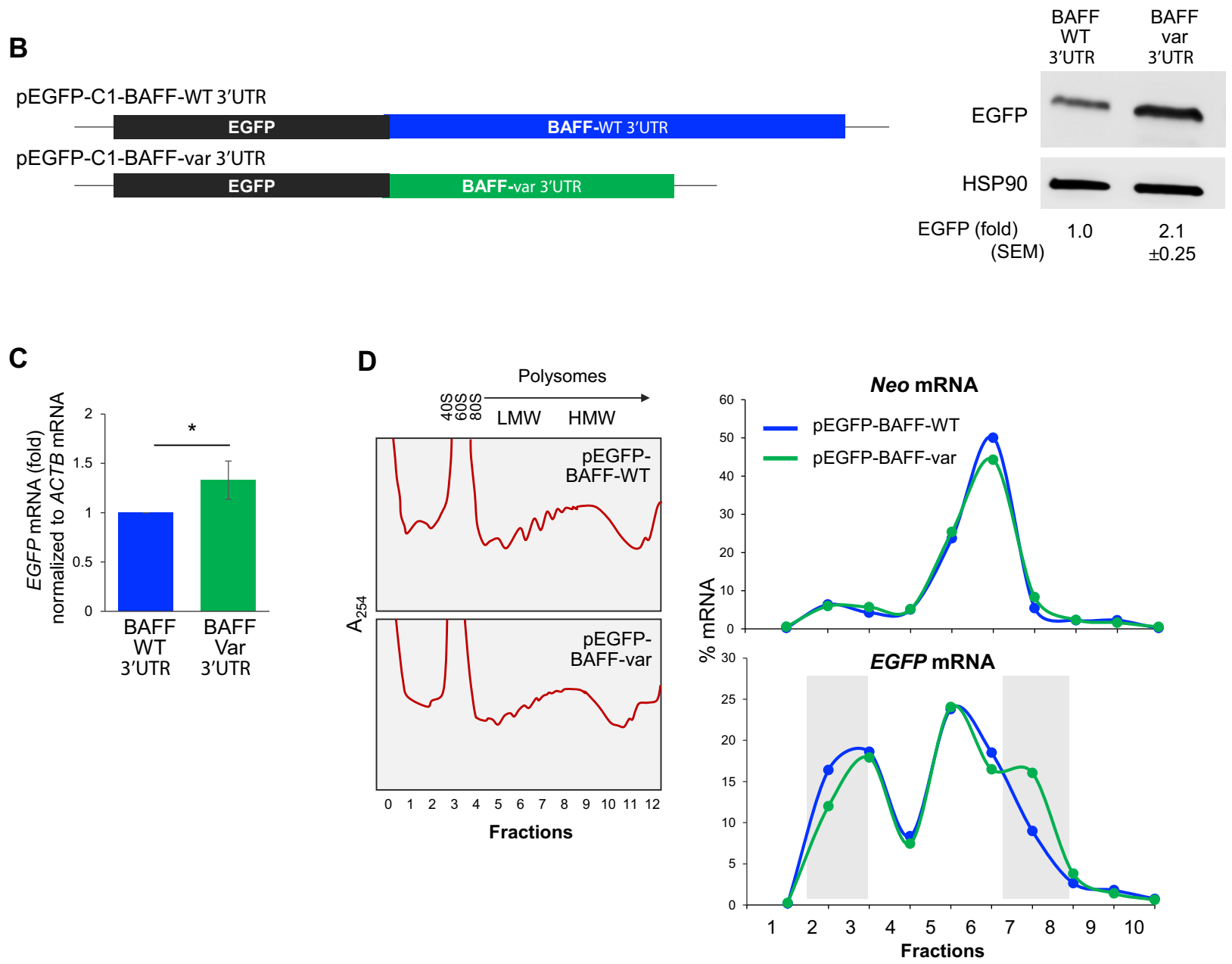

Figure 1. Differential regulation of BAFF from BAFF-var and BAFF-WT mRNAs. (A) Top, schematic of the TNFSF13B gene, polyadenylation site (PA), and alternative polyadenylation site (APA) generated due to a polymorphic insertion-deletion. Bottom, mRNAs generated from TNFSF13B according to polyadenylation site usage [BAFF-WT mRNA (blue) and $B A F F$-var mRNA (green)], as well as relative circulating soluble BAFF proteins levels measured from the Sardinia cohort by ELISA in plasma from individuals homozygous for $B A F F$-WT (1280) or for BAFF-var (272) (8). (B) Left, reporter constructs derived from pEGFP-C1, carrying the BAFF-WT (top) and BAFF-var 3'UTR (bottom). Right, $24 \mathrm{~h}$ after transfecting HeLa cells with the constructs, western blot analysis was used to assess EGFP levels; HSP90 was assessed as a loading control. Signals were quantified using ImageJ. (C) EGFP mRNA levels in each transfection group, as measured by RT-qPCR analysis. (D) Left, $24 \mathrm{~h}$ after transfecting HeLa cells with the constructs in panel (B), cytoplasmic lysates were fractionated through sucrose gradients, and RNA was extracted from fractions 1 and 2 (free RNA/RNPs), fractions 3 and 4 [ribosomal subunits $(40 \mathrm{~S}, 60 \mathrm{~S})$ and monosomes $(80 \mathrm{~S})]$, fractions 5-7 [low-molecular-weight (LMW) polysomes], and fractions 8-10 [high-molecular weight (HMW) polysomes]; fractions 11 and 12 typically had no detectable RNA and were discarded. Right, relative distribution of Neo mRNA, expressed from the pEGFP expression vectors and included as a control mRNA, and the EGFP-BAFF-WT and EGFP-BAFF-var mRNAs, expressed from the respective vectors, as measured by RT-qPCR analysis and represented as the percentage of total mRNA in the gradient. Shaded rectangles highlight regions of rightward shift in the distribution for $E G F P-B A F F$-var mRNA on sucrose gradient compared to EGFP-BAFF-WT mRNA. Results shown are representative of three independent experiments. In (B, C), data are the means and standard errors of the means (SEM) of three or more independent experiments $(* P<0.05$; significance was determined by Student's $t$-test). 
BAFF-WT (blue) or BAFF-var (green): plasmids pEGFPC1-BAFF-WT 3'UTR and pEGFP-C1-BAFF-var 3'UTR (Figure 1B). The impact of each $3^{\prime} \mathrm{UTR}$ was tested by transfecting HeLa cells with each reporter construct; $24 \mathrm{~h}$ later, western blot analysis of EGFP levels revealed that greater than twofold higher EGFP was expressed from the variant reporter (pEGFP-C1-BAFF-var 3'UTR) (Figure $1 \mathrm{~B}$, right). These differences did not completely arise from EGFP mRNA levels, assessed by reverse transcription (RT) followed by quantitative (q)PCR analysis (Figure 1C), as the variant transcript (EGFP-BAFF-var $3^{\prime} U T R$ mRNA) was only 1.3 -fold more abundant than the WT transcript (EGFP-BAFF-WT 3'UTR mRNA). These results agreed with our earlier findings (8) suggesting that BAFF production increased through both higher mRNA abundance and enhanced translation. A numerical measure of translation status known as the 'translation index' is calculated as the ratio of differences in protein levels relative to differences in the corresponding mRNA levels. For the reporters studied in Figures $1 \mathrm{~B}$ and $\mathrm{C}$, the abundance ratio of $\mathrm{mRNAs}$ produced from each vector (EGFP-BAFF-var 3'UTR mRNA relative to $E G F P-B A F F-W T 3^{\prime} U T R$ mRNA) is 1.3 , while the ratio of EGFP proteins produced from each vector is 2.1. Thus, the relative translation index for these two constructs, 1.6, is consistent with the notion that translation is enhanced.

To test directly the possibility of regulated translation, we assessed the rate of translation of each chimeric mRNA by measuring the sizes of polysomes associated with each mRNA. Twenty-four hours following transfection, cytoplasmic preparations from transfected HeLa cells were fractionated through sucrose gradients, yielding fractions that lacked ribosome components (fractions 1 and 2), fractions with ribosome subunits $40 \mathrm{~S}$ and $60 \mathrm{~S}$, as well as single monosomes (80S) (fractions 3 and 4), and fractions with low-molecular-weight (LMW) and high-molecular-weight (HMW) polysomes (fractions 5-7 and 8-10, respectively). As shown in Figure 1D, there were no major differences in polysome profiles between the transfection groups. After isolating RNA from each fraction, the levels of $E G F P$ $B A F F$-var and EGFP-BAFF-WT mRNAs were measured by RT-qPCR analysis and represented as a percent of that mRNA in each fraction relative to the total amount of mRNA in that sample. As shown in Figure 1D (right) and Supplementary Figure S4B, there was a modest but consistent shift between the distributions of the two chimeric mRNAs: compared to the distribution of $E G F P-B A F F-W T$ mRNA, EGFP-BAFF-var mRNA shifted rightward, revealing that it associated with larger polysomes (a shift that was consistent with more active translation), and exhibited a second peak at fraction 7 , indicating that a subset of larger polysomes formed on the EGFP-BAFF-var mRNA. By contrast, no such shifts were observed when measuring the levels of control neomycin-resistance (Neo) mRNA, expressed constitutively from the $\mathrm{pEGFP-C1}$ reporter backbone, confirming that the two transfection groups shared similar rates of global protein translation, and further supporting the notion that translation of EGFP from the EGFP-BAFF-var chimeric mRNA was selectively enhanced. The relative distribution of the transcript encoding the housekeeping protein $\beta$-Actin ( $A C T B$ mRNA) was also comparable between the transfection groups (not shown). Together, these results indicate that compared to the $3^{\prime} \mathrm{UTR}$ of $B A F F$ - $W T$ mRNA, the shorter $3^{\prime} \mathrm{UTR}$ of $B A F F$-var mRNA conferred more robust translation of BAFF.

\section{NF90 preferentially binds $B A F F-W T 3^{\prime}$ UTR}

To search for factors that might modulate BAFF translation, we employed an affinity pulldown strategy to identify RBPs interacting with $B A F F 3^{\prime} \mathrm{UTR}$. Biotinylated $B A F F$ $3^{\prime} \mathrm{UTR}$ was synthesized in vitro (Materials and Methods) and incubated with whole-cell extracts prepared from THP1 cells, an immortalized monocytic leukemia line chosen because monocytes and macrophages are the main producers of BAFF. Ribonucleoprotein (RNP) complexes were pulled down using streptavidin beads and the interacting proteins were identified by mass spectrometry (MS) analysis (Figure $2 \mathrm{~A}$, left ; control beads were analyzed in parallel. Among the many proteins detected in the pulldown materials, the most enriched RBP was the interleukin enhancer-binding factor 3 (ILF3, also known as nuclear factor 90, NF90) (Figure 2A, table). Following validation by western blot analysis of several RBPs identified in the MS screen, including $\mathrm{HuR} / \mathrm{ELAVL1}, \mathrm{AGO} 2$, and CELF1, NF90 was found to display most extensive binding to $B A F F 3^{\prime}$ UTR (Figure 2B). GAPDH 3'UTR, previously found to bind NF90 (not shown), was included as a positive control

Finer mapping of the region(s) of interaction of NF90 with $B A F F 3^{\prime}$ UTR was conducted by preparing biotinRNA segments spanning the $B A F F 3^{\prime} \mathrm{UTR}$. After incubation with THP-1 whole-cell lysates, pulldown analysis using streptavidin beads followed by NF90 western blot analysis (including the GAPDH $3^{\prime} \mathrm{UTR}$ RNA as a positive control) revealed that NF90 associated most avidly with segment 6, which spanned the APA; GAPDH 3'UTR was again included as a positive control. Following the division of RNA 6 into fragments $6 \mathrm{a}$ and $6 \mathrm{~b}$, fragment $6 \mathrm{~b}$ was found to show stronger association. Interestingly, this fragment is located immediately downstream of the APA, supporting the notion that NF90 selectively binds the $B A F F-W T$ mRNA isoform (Figure 2C).

To gain independent evidence that endogenous NF90 interacts with endogenous $B A F F$ mRNA, we carried out RNP immunoprecipitation (RIP) analysis in THP-1 cells employing an anti-NF90 antibody and $\mathrm{IgG}$ in control IP reactions; NF90 levels in each IP sample were monitored by western blot analysis (Figure 2D). RNA was then extracted and subjected to RT-qPCR analysis using $B A F F$ mRNAspecific primers. As shown in Figure 2D, $B A F F$ mRNA associated with endogenous NF90 in THP-1 cells, as demonstrated by the enrichment in $B A F F$ mRNA in NF90 RIP samples compared with IgG IP (Figure 2D); enrichments were normalized to the levels of ubiquitin $\mathrm{C}(U B C)$ mRNA in each IP reaction.

Additional evidence that NF90 bound the long $B A F F$ mRNA was obtained using lymphoblastoid cells generated from donors from the SardiNIA cohort who were confirmed to have either $B A F F-W T$ or $B A F F$-var genotypes. RIP analysis in these cells revealed that endogenous NF90 associated with $B A F F$ mRNA significantly more in BAFFWT cells (which expressed only $B A F F-W T$ mRNA) com- 
A

\begin{tabular}{|c|c|c|c|c|}
\hline \multicolumn{5}{|l|}{ BAFF-WT mRNA $\quad$ BAFF-WT 3'UTR mRNA } \\
\hline & Protein & Description & $\begin{array}{l}\Sigma \\
\text { Coverage }\end{array}$ & $\begin{array}{l}\text { \# PSM } \\
\text { BAFF 3'UTR }\end{array}$ \\
\hline biotin-l & ILF3 (NF90) & Interleukin enhancer-binding factor 3 & 71.70 & 168 \\
\hline & PABP4 & Polyadenylate-binding protein 4 & 56.83 & 158 \\
\hline & PABP1 & Polyadenylate-binding protein 1 & 53.14 & 90 \\
\hline e-cel & ILF2 & Interleukin enhancer-binding factor 2 & 75.38 & 55 \\
\hline & E2AK2 & Interferon-Inducible EIF2a Kinase & 33.03 & 36 \\
\hline & ROA3 & Heterogeneous nuclear ribonucleoprotein A3 & 37.30 & 14 \\
\hline & MATR3 & Matrin-3 & 50.41 & 34 \\
\hline & DI3L2 & DIS3-like exonuclease 2 & 47.23 & 41 \\
\hline & AGO2 & Protein argonaute-2 & 25.38 & 13 \\
\hline & $\mathrm{PRC} 2 \mathrm{C}$ & Protein PRRC2C & 19.27 & 27 \\
\hline & ELAVL1 & ELAV-like protein 1 & 39.57 & 9 \\
\hline & PABP2 & Polyadenylate-binding protein 2 & 31.37 & 12 \\
\hline Mass Spectrometry & U2AF2 & Splicing factor U2AF 65-kDa subunit & 23.37 & 5 \\
\hline and We & CELF2 & CUGBP Elav-like family member 2 & 21.46 & 4 \\
\hline & CELF1 & CUGBP Elav-like family member 1 & 19.14 & 1 \\
\hline
\end{tabular}

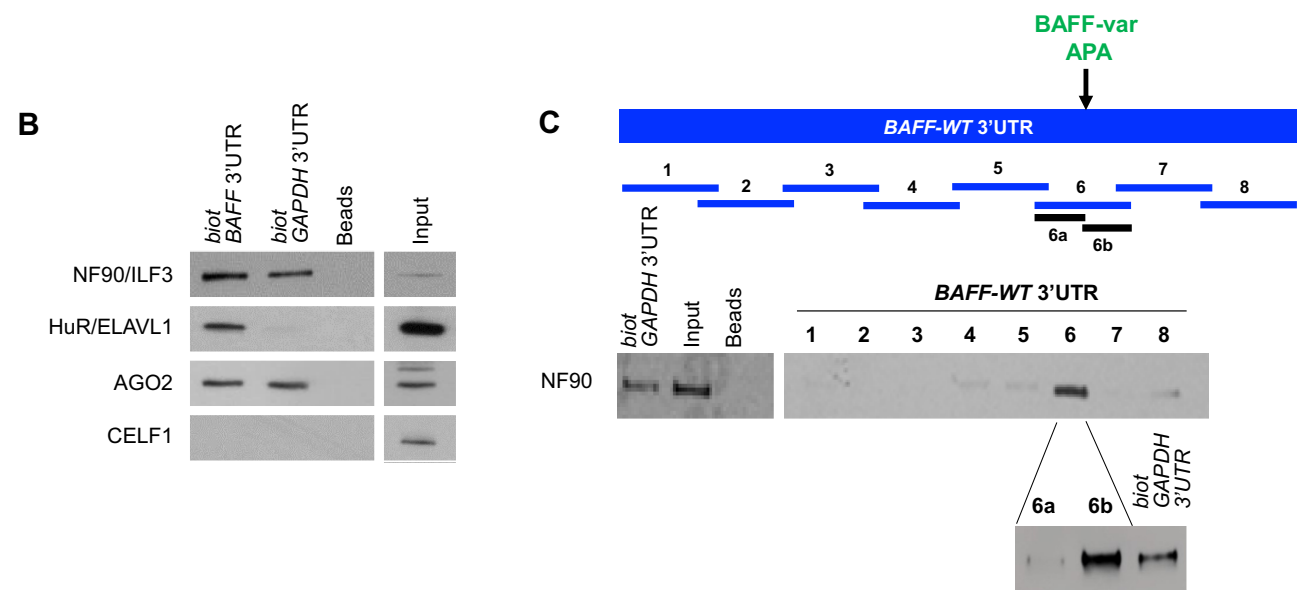

D

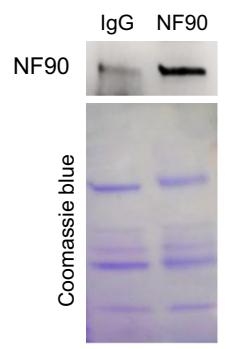

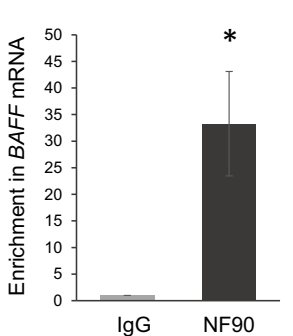

$\mathbf{E}$

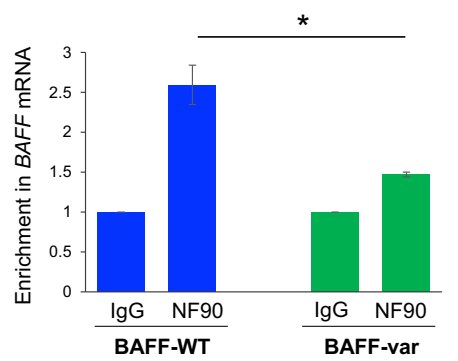

Figure 2. NF90 binds distal to the APA in $B A F F 3^{\prime} \mathrm{UTR}$. (A) Left, schematic representation of $B A F F-W T$ mRNA and the biotinylated full-length $3^{\prime} \mathrm{UTR}$ prepared for pulldown analysis using streptavidin beads to identify THP-1 proteins with affinity for $B A F F 3^{\prime} \mathrm{UTR}$. After pulldown, the material was washed and the associated proteins were size-fractionated by SDS-PAGE and analyzed by mass spectrometry (MS) analysis (right, Materials and Methods, Supplementary Table S1). Those RBPs enriched in biotin- $B A F F 3^{\prime} \mathrm{UTR}$ relative to beads are listed, along with the number of peptides recovered for each RBP in the MS analysis (right). (B) Western blot analysis was performed to verify the specific association of biotin-BAFF $3^{\prime} \mathrm{UTR}$ with several RBPs (NF90/ILF3, HuR/ELAVL1, AGO2, CELF1) identified by MS analysis. (C) To identify the region of NF90 binding on $B A F F$ mRNA, small biotin-RNA fragments spanning the $3^{\prime}$ UTR were generated (fragments 1 through 8), incubated with THP-1 lysate, and subjected to pulldown followed by western blot analysis to identify the RNAs that associated with NF90. Fragment 6 was subdivided into fragments $6 \mathrm{a}$ and $6 \mathrm{~b}$, and each was assayed similarly for interaction with NF90. (D) The association of NF90 with BAFF mRNA was tested by ribonucleoprotein immunoprecipitation (RIP) analysis using antiNF90 antibody. Left, after IP using anti-NF90 or IgG antibodies, the presence of NF90 in the IP material was assayed by western blot analysis (top), and equal loading was monitored by staining with Coomassie blue (bottom). Following RNA extraction, the enrichment of $B A F F$ mRNA in NF90 IP samples was assessed by RT-qPCR analysis using $B A F F$-specific primers, normalized to the levels of $U B C$ mRNA in each sample, and plotted relative to background $B A F F$ mRNA levels in the IgG IP samples. (E) RIP analysis was subsequently performed in whole-cell lysates of LCL cells prepared from donors who were homozygotic for either $B A F F-W T$ or $B A F F$-var. NF90 IP and RT-qPCR detection of $B A F F$ mRNA were performed as described in panel 2D. Data in $(\mathrm{B}, \mathrm{C})$ are representative of at least 3 experiments; data in $(\mathrm{D}, \mathrm{E})$ are the means and SEM from at least 3 independent experiments $(* P<$ 0.05 ; significance was determined by Student's $t$-test). 
pared with BAFF-var cells, which expressed high levels ( $\sim 70 \%$ for individuals homozygous for BAFF-var) of the shorter, BAFF-var mRNA (Figure 2E). Only BAFF-WT cells reached the 2-fold enrichment that is generally used as the cut-off to identify bona fide interactions. BAFF-var cells showed a 1.5 -fold enrichment likely the result of (i) weak binding of the proximal half of the $B A F F$ mRNA (fragments $1-5$ in Figure 2C) and (ii) the presence in BAFF-var cells of a mixture of $B A F F-W T$ mRNA $(\sim 30 \%)$ and $B A F F$ var mRNA $(\sim 70 \%)$.

Taken together, these data indicate that NF90 preferentially binds the $3^{\prime} \mathrm{UTR}$ of the $B A F F-W T$ mRNA over the $B A F F$-var mRNA.

\section{NF90 suppresses BAFF translation}

To investigate if NF90 regulates BAFF production, we generated a THP-1 line (pooled population) stably expressing NF90 shRNA, along with a scrambled shRNA control line. Interestingly, the reduction in NF90 levels in THP-1 cells led to higher BAFF protein production (Figure $3 \mathrm{~A}$ ) and higher soluble BAFF (not shown); no significant changes in $B A F F$ mRNA levels were observed after lowering NF90 abundance in THP-1 cells (Figure 3B). The hypothesis that NF90 might reduce $B A F F$ mRNA translation without affecting its stability was in agreement with earlier reports that NF90 represses the translation of several cytokines without altering the half-lives of the respective mRNAs $(13,15)$.

To test this hypothesis, we monitored the distribution of $B A F F$ mRNA in polysome gradients prepared from control and NF90-silenced THP-1 cells. As shown in a representative experiment (Figure 3C, left), global polysome profiles were similar between the two cell lines. However, the distribution of $B A F F \mathrm{mRNA}$ along the gradient, as determined by RT-qPCR analysis, shifted rightward when NF90 was silenced, yielding relatively lower $B A F F$ mRNA levels in unbound fractions (e.g. fractions 2 and 3 ) and relatively higher in polysomal fractions (e.g. fractions 6 and 7). This pattern was indicative of $B A F F$ mRNA forming larger polysomes and hence being more actively translated (Figure $3 \mathrm{C}$, right). $A C T B$ mRNA, encoding the housekeeping protein $\beta$-Actin, was found distributed with comparable patterns regardless of NF90 status (Figure 3C, right and Supplementary Figure $\mathrm{S} 4 \mathrm{C}$ ). To validate this result using a different approach, THP-1 cells were transiently transfected with siRNA directed to NF90 or control siRNA; analysis of polysomes from these cells was also consistent with NF90 suppressing $B A F F$ mRNA translation (Supplementary Figure S1A-D).

To further investigate the regulation of BAFF production by NF90, and to address whether NF90 affected differentially the translation of $B A F F-W T$ and $B A F F$-var $\mathrm{mR}$ NAs, we studied the influence of NF90 on the production of EGFP from reporter constructs bearing $B A F F-W T$ or $B A F F$-var $3^{\prime}$ UTRs (Figure 1B) in THP-1 cells with NF90 stably silenced (described in Figure 3A). Twenty-four hours after transfection of THP-1 cells with the EGFP reporters shown in Figure 3D, the levels of EGFP produced were assessed by western blot analysis. In NF90-silenced cells, the levels of EGFP synthesized from a chimeric mRNA bearing the $B A F F-W T 3^{\prime} \mathrm{UTR}$ were $>2$-fold higher than those synthesized from cells expressing normal NF90 levels or from cells expressing BAFF-var 3'UTR (or no BAFF sequences, 'Vector') (Figure 3D, right). These findings indicate that NF90 represses BAFF translation by acting upon the full-length $B A F F 3^{\prime} \mathrm{UTR}$ allele (WT) but does not repress BAFF production from the shorter $B A F F 3^{\prime} \mathrm{UTR}$ allele (var).

To test directly whether NF90 regulates BAFF production, we generated a vector that expressed a $B A F F 3^{\prime} \mathrm{UTR}$ lacking the NF90 binding site [pEGFP-BAFF $3^{\prime}$ UTR $(\triangle \mathrm{NF} 90)$ (Figure $3 \mathrm{E})]$. $B A F F 3^{\prime} \mathrm{UTR} \mathrm{WT}$ and $\triangle \mathrm{NF90}$ reporters were transfected into THP-1 cells in which NF90 was stably silenced, and EGFP expression was analyzed 24 $\mathrm{h}$ later. As shown in Figure 3E, EGFP production from $E G F P-B A F F 3^{\prime} U T R(\triangle N F 90) \mathrm{mRNA}$ failed to respond to change in NF90 abundance, supporting the notion that NF90 binding to $B A F F 3^{\prime} \mathrm{UTR}$ was required to regulate BAFF expression.

Collectively, our results indicate that NF90 represses BAFF production and secretion by lowering $B A F F$ mRNA translation. They further reveal that NF90 actively represses the translation of full-length $B A F F$ mRNA ( $B A F F$ $W T$ mRNA), but not the short variant (BAFF-var mRNA) lacking the site of NF90 binding.

\section{NF90 represses BAFF translation by promoting the associa- tion of miR-15a with $B A F F$ mRNA}

We hypothesized that the translational repression elicited by NF90 might be linked to the previously identified inhibition of BAFF production by miR-15a (8). Given that there is one miR-15a site on $B A F F$-var mRNA and two on BAFF-WT mRNA (Figure 4A), BAFF-var mRNA was more abundant constitutively and produced higher levels of BAFF protein, as previously discovered by cotransfection of HeLa cells with miR-15a and pmirGLO-BAFF $3^{\prime}$ UTR constructs (8). Indeed, only $B A F F-W T$ mRNA associated with NF90 also had a miR-15a site downstream of the APA (Figure 4A). Thus, by $48 \mathrm{~h}$ after transfecting THP-1 cells (which bear two $B A F F-W T$ alleles and hence only express $B A F F-W T$ mRNA) with miR-15a mimic to enhance miR15a levels (Supplementary Figure S1E), BAFF production and secretion were reduced, while transfection of a miR-15a antagomiR [antisense (AS) miR-15a] to suppress miR-15a function increased BAFF production and secretion (Figure $4 \mathrm{~B}$ and $\mathrm{C}$ ). miR-15a overexpression did not affect $B A F F$ mRNA levels significantly (Figure 4D). Interestingly, overexpressing miR-15a reduced NF90 levels while antagonizing miR-15a elevated NF90 levels (Figure 4B).

To test if miR-15a and NF90 actions were functionally linked, we first investigated, in THP-1 cells, if the microRNA-binding AGO protein family associated with $B A F F$ mRNA differently depending on NF90 levels. Since MS analysis (Table in Figure 2A) indicated that AGO2, but not other AGO proteins, bound $B A F F$ mRNA, we concentrated our analysis on AGO2. The interaction of other AGO proteins with $B A F F \mathrm{mRNA}$, and the possible regulation of BAFF expression by other AGO proteins was analyzed (Supplementary Figures S2 and S3, Supplementary text). We observed interaction between $\mathrm{AGO} 2$ and NF90, as determined by co-IP analysis in THP-1 cells (Supplementary Figure S2C), and further established that the interac- 
A

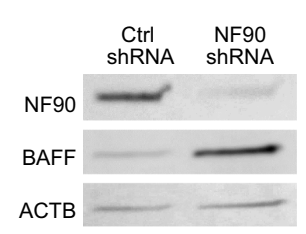

C

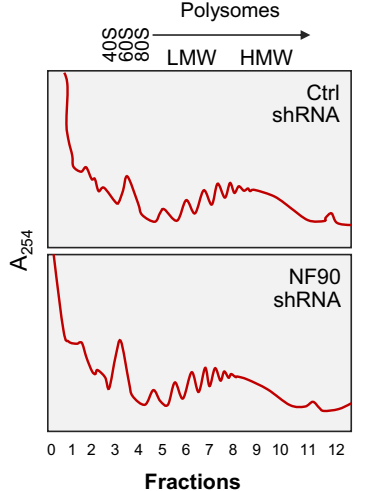

Fractions
B

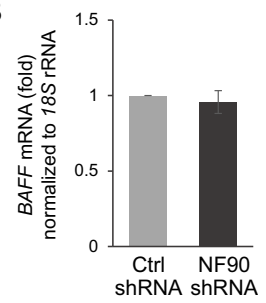

D

pEGFP-C1 (Vector)

pEGFP-C1-BAFF-WT 3'UTR

EGFP
EGFP-C1-BAFF-var 3'UTR
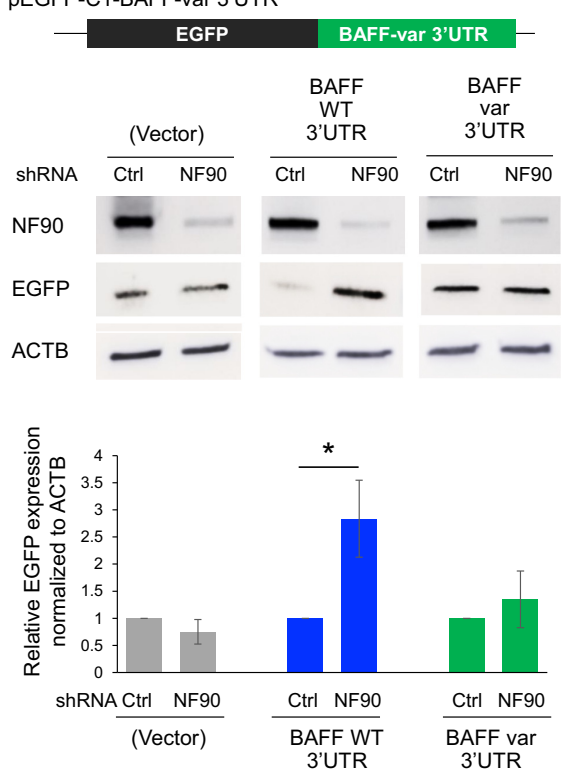

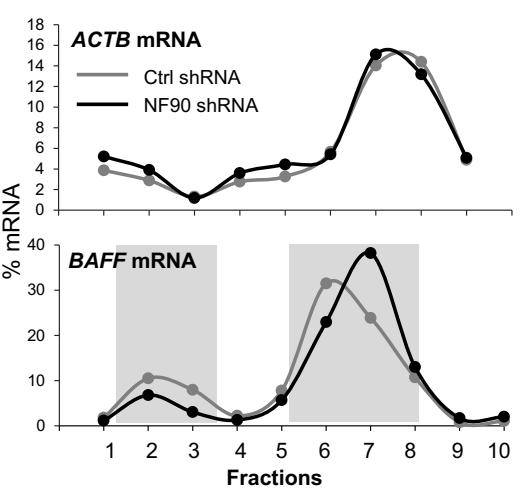

E
pEGFP-C1-BAFF-WT 3'UTR

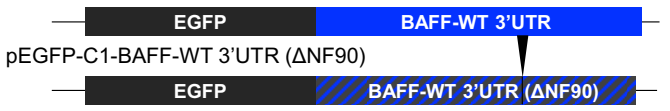

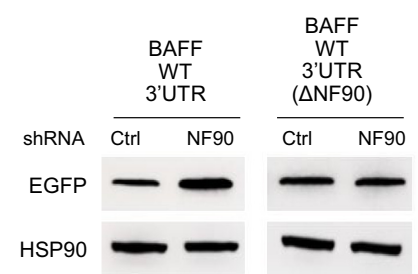

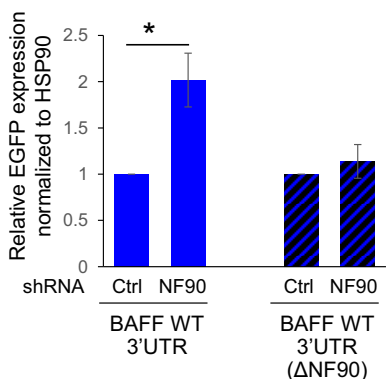

Figure 3. NF90 represses BAFF translation. (A, B) Western blot analysis (A) and $B A F F$ mRNA (B) produced by THP-1 cell populations with stably reduced NF90 levels (NF90 shRNA) and control cells (Ctrl shRNA). (C) The relative distribution of $B A F F$ mRNA (and $A C T B$ mRNA, encoding the housekeeping protein $\beta$-Actin) in polysomes prepared from the THP-1 populations described in (A) was assessed by RT-qPCR analysis as described in Figure 1D. Shaded rectangles highlight the rightward shift in the distribution of $B A F F$ mRNA in sucrose gradients in the NF90 shRNA group compared to the Ctrl shRNA group. (D) Schematic of the EGFP reporter constructs bearing the $B A F F$-WT $3^{\prime} \mathrm{UTR}$, the $B A F F$-var $3^{\prime} \mathrm{UTR}$, or no $3^{\prime} \mathrm{UTR}$ (Vector) used for reporter analysis. Twenty-four hours after transfecting the THP-1 cells described in panel (A) with each EGFP vector, EGFP expression levels were assessed by western blot analysis (top). EGFP signals from three experiments were analyzed using imageJ and plotted relative to Ctrl shRNA in each transfection group (bottom). (E) Schematic of EGFP reporter constructs bearing the $B A F F-W T 3^{\prime} \mathrm{UTR}$, or the $B A F F-W T 3^{\prime} U T R(\triangle N F 90) 3^{\prime} \mathrm{UTR}$. Twenty-four hours after transfecting THP-1 cells as described in (A) with each vector, EGFP expression levels were assessed by western blot analysis. EGFP signals from three experiments were quantified using imageJ and plotted relative to Ctrl shRNA in each transfection group. Data in (B, D, E) are the means and SEM from at least 3 independent experiments $\left({ }^{*} P<0.05\right.$; significance was determined by Student's $t$-test). 
A

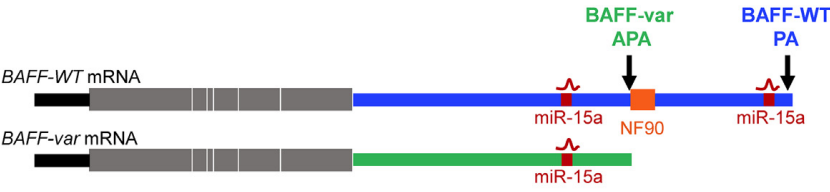

B

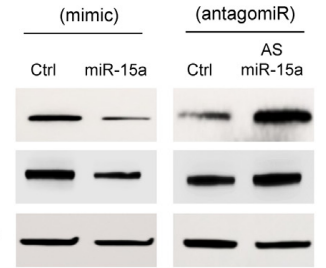

E

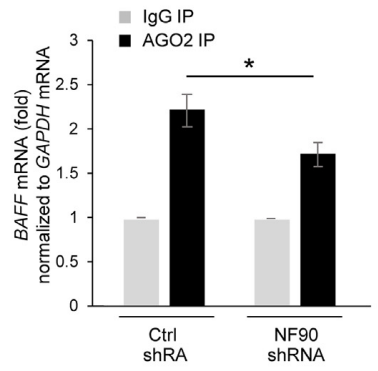

C

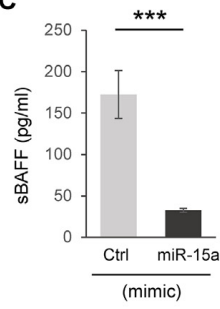

$\mathbf{F}$

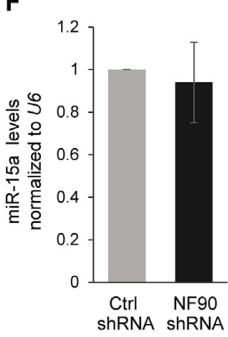

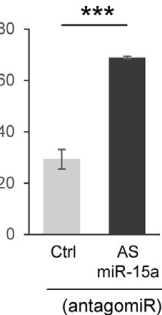

H
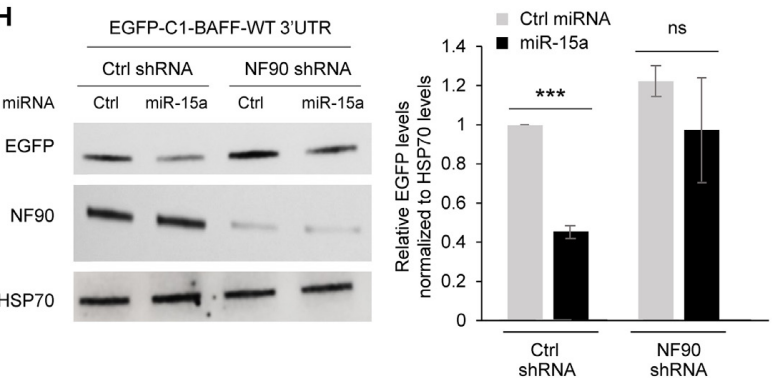

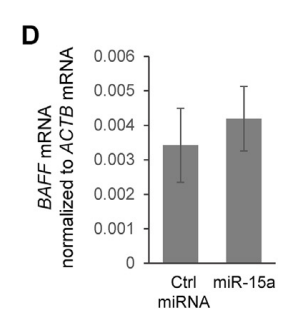

G

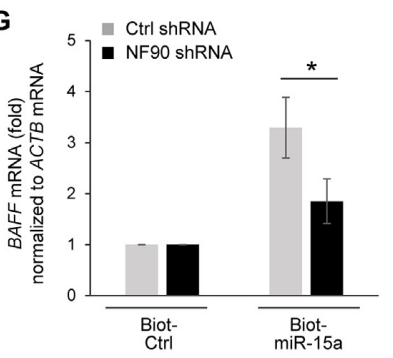

I

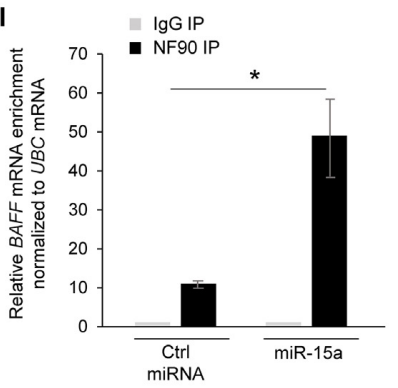

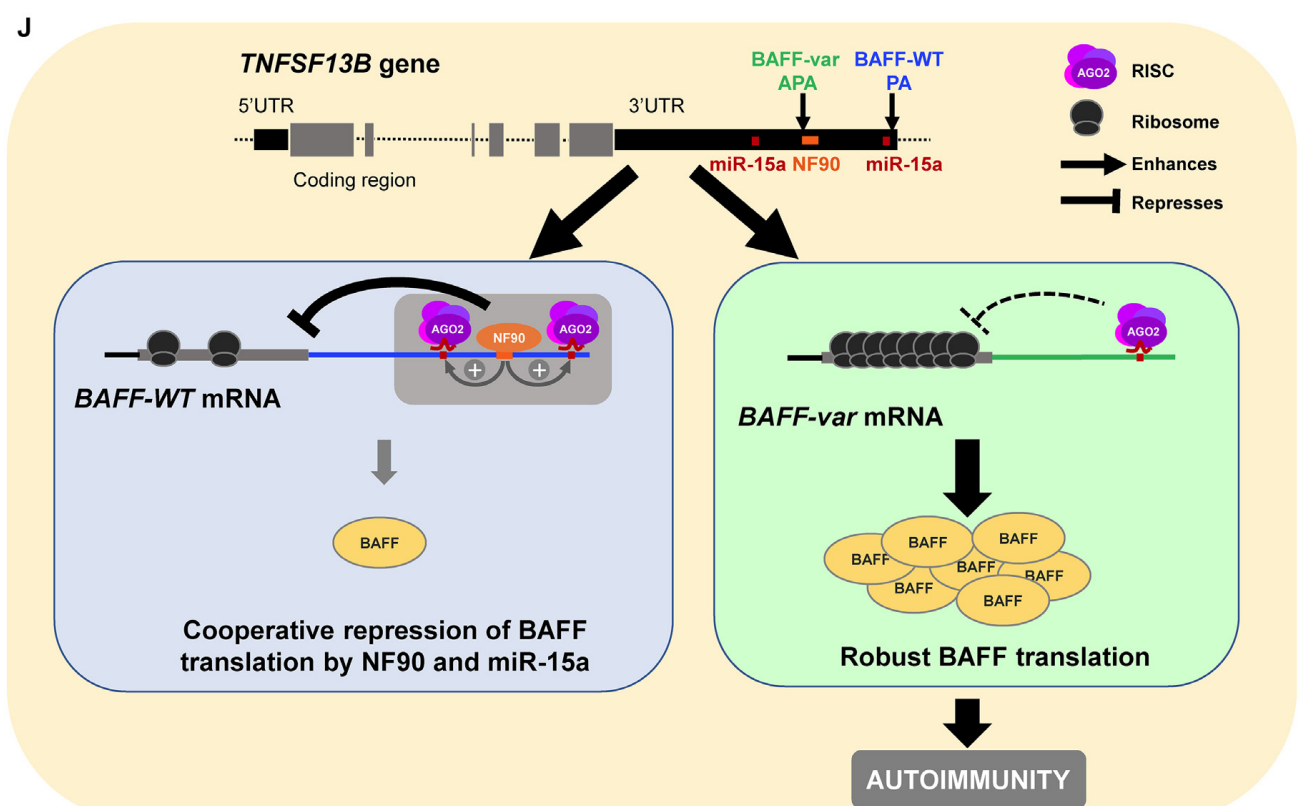

Figure 4. NF90 cooperates with miR-15a to suppress BAFF translation. (A) Schematic of BAFF-WT and BAFF-var mRNAs, with the location of miR$15 \mathrm{a}$ and NF90 indicated. (B, C) The levels of BAFF, NF90 and sBAFF produced by THP-1 cells were assessed by western blot analysis (B) or ELISA (C) 
tion was RNA-dependent (not shown). As shown in Figure 4E, in THP-1 cells constitutively expressing lower NF90 levels, the interaction of $\mathrm{AGO} 2$ with $B A F F \mathrm{mRNA}$, as assessed by RIP analysis using an anti-AGO2 antibody (Materials and Methods), was lower than in control cells, indicating that NF90 increased the interaction of microRNAAGO2 complexes with $B A F F$ mRNA. Control shRNA and NF90 shRNA populations of THP-1 cells had similar levels of miR-15a (Figure 4F).

Next, we assayed whether the binding of miR-15a to $B A F F$ mRNA was altered in cells with reduced NF90 levels. Forty-eight hours after transfection of biotinylated miR$15 \mathrm{a}$, lysis, and pulldown using streptavidin-coated beads, significantly more $B A F F$ mRNA was recovered in THP-1 cells with normal NF90 levels (Ctrl shRNA) than in THP1 cells with silenced NF90 (Figure 4G). Furthermore, cells in which NF90 was silenced displayed an impaired ability to lower BAFF production by miR-15a, as assessed by measuring EGFP production from the reporter construct pEGFP-C1-BAFF-WT 3'UTR (Figure 4H). As expected, baseline production of EGFP from this construct increased in the NF90 shRNA group, since reducing NF90 levels de-repressed EGFP production driven by the $B A F F$ $W T 3^{\prime} \mathrm{UTR}$. A direct role of miR-15a in regulating $B A F F$ mRNA was further confirmed by using a pEGFP-BAFF $3^{\prime}$ UTR construct in which the miR-15a binding sites were mutagenized; this vector was refractory to the repressive effects of miR-15a (Supplementary Figure S4A). Interestingly, NF90 binding to $B A F F \mathrm{mRNA}$ increased when miR15a was overexpressed in THP-1 cells (Figure 4I), supporting the notion that miR-15a facilitated $B A F F$ mRNA-NF90 interaction (Figure 4I).

Taken together, these findings indicate that the presence of NF90 contributed to the interaction of $B A F F-W T$ mRNA with miR-15a to elicit BAFF repression (Figure 4J).

\section{DISCUSSION}

We have identified NF90 as an RBP that associates with the most common $B A F F$ mRNA ( $B A F F-W T$ mRNA) and reduces $\mathrm{BAFF}$ translation. The shorter variant $B A F F$-var mRNA lacks the NF90 site and is thus more actively translated, leading to enhanced BAFF production in THP1 cells (Figure 4J). The differential impact of NF90 on BAFF production recapitulates the effects reported for miR-15a, which selectively repressed $B A F F-W T$ mRNA but not $B A F F$-var mRNA (8). Importantly, the interaction of miR-15a with $B A F F-W T$ mRNA was enhanced by NF90, suggesting a cooperative mode of action whereby NF90 binding to $B A F F$ mRNA facilitates miR-15a recruitment and translational repression of $B A F F$ mRNA (Figure $4 \mathrm{~J}$ ). Interestingly, this cooperation may be further solidified by the miR-15a-promoted interaction of NF90 with $B A F F$ mRNA (Figure 4I). The reduction of NF90 levels by miR15a (Figure 4B) may represent a compensatory mechanism to keep the suppression of BAFF production by miR-15a in check; additional investigation is needed to fully understand this regulatory loop.

The BAFF-WT $3^{\prime} \mathrm{UTR}$ is longer than the BAFF-var 3'UTR. Long 3'UTRs generally enable additional regulation of the mRNA, influencing both their stability and translational efficiency (16). The presence of additional regulatory motifs drive the assembly of RNP complexes that regulate mRNA abundance, as well as the time and location of protein production (17). Here, the cooperative binding of miR-15a and NF90 to $B A F F-W T$ mRNA that suppressed BAFF translation was lost in the shorter $B A F F$-var mRNA. Additional studies are needed to identify other possible $c i s$ elements driving BAFF expression through the short and long 3'UTRs.

There are several reports of coordinated actions of RBPs and microRNAs on a given mRNA, as reviewed $(18,19)$. This coordination can occur sequentially (if the microRNA and the RBP bind one after the other) or simultaneously (if they bind at distant or proximal locations at the same time). Proximal interactions may be competitive, if the binding of one displaces or prevents the binding of the other, or cooperative, if one enhances the binding of the other, as observed here for NF90 and miR-15a. Functionally, both factors may have a negative impact on an mRNA (e.g. both reduce stability or translation), or both may have a positive effect; in each case, their joint impacts might then be additive or synergistic. If the factors have opposite effects, then the net impact on the target mRNA can be more complex and will depend on their relative availabilities at a given place and time.

However, only a handful of cooperative interactions between RBPs and microRNAs affecting mRNA fate have been reported. For example, binding of RBP PUM1 to target $p 27$ mRNA opened the local RNA secondary structure, allowing miRNAs miR-221 and miR-222 to bind and suppress the target mRNAs (20). Similarly, binding of RBP

$48 \mathrm{~h}$ after overexpressing miR-15a by transfecting a miR-15a mimic (left) or neutralizing miR-15a by transfecting a miR-15a antagomir ( right). (D) BAFF mRNA levels in THP-1 cells transfected with miR-15a mimic as in panel (B) were measured 48 h later by RT-qPCR analysis. (E) AGO2 RIP analysis was performed in cells expressing normal (Ctrl shRNA) or reduced (NF90 shRNA) NF90 levels to identify AGO2-bound BAFF mRNA. (F) The steady-state levels of miR-15a in the cells described in panel (E) were assessed by RT-qPCR analysis. (G) The cells described in (E) were transfected with biotinylated miR-15a or a biotinylated control RNA; $48 \mathrm{~h}$ later, cells were lysed and the levels of $B A F F$ mRNA enrichment in the biot-miR-15a pulldown were assessed by RT-qPCR analysis. (H) THP-1 cells described in (E) were co-transfected with a chimeric reporter construct expressing $E G F P$ - $B A F F$ - $W T 3^{\prime} U T R$ mRNA and either Ctrl miRNA or miRNA-15a mimic; $24 \mathrm{~h}$ later, EGFP expression levels were assessed by western blot analysis (left). EGFP signals were quantified using ImageJ and plotted (right). (I) Forty-eight hours after transfecting THP-1 cells with Ctrl miRNA or miR-15a mimic, the interaction of NF90 with $B A F F$ mRNA was assessed by RIP followed by RT-qPCR analysis. (J) Model summarizing the results of this study. The wild-type TNFSF13B gene uses the constitutive polyadenylation site (blue, PA) to give rise to $B A F F$ - $W T$ mRNA (left), bearing a long $3^{\prime} \mathrm{UTR}$ with two miR-15a sites and an NF90 binding site; the cooperative actions of these factors contribute to maintaining low BAFF translation levels and overall BAFF production. The variant $T N F S F 13 B$ gene uses with high frequency an upstream polyadenylation site (green, APA) that gives rise to a shorter transcript, $B A F F$-var mRNA ( $\mathrm{might}$ ), bearing a shorter $3^{\prime}$ UTR that lacks the distal miR-15a site and the NF90 binding site; consequently, BAFF-var mRNA is translated at aberrantly higher levels and results in the production of higher BAFF in individuals bearing this variant sequence (8). In $(\mathrm{C}, \mathrm{D}, \mathrm{G}, \mathrm{H}, \mathrm{I})$, the data represent the means and $\mathrm{SEM}$ from three independent experiments $\left({ }^{*} P<0.05,{ }^{*} * P<0.001\right.$; significance was determined by Student's $t$-test). 
HuR to a long noncoding RNA (lincRNA-p21) promoted let-7 association with lincRNA-p21 and triggered lincRNAp21 degradation (21). In this regard, the discovery that NF90 promoted the suppressive actions of miR-15a represents a novel mechanism of action for NF90, with potentially important implications for immune gene expression programs.

Generated by alternative splicing of the ILF3 gene, NF90 is a ubiquitous protein that is localized primarily in the nucleus but is also present in the cytoplasm. Although NF90 is capable of binding DNA and regulating transcription, as reviewed by Castella et al. (22), its ability to bind singleand double-stranded RNA has established NF90 as a major regulator of mammalian and viral RNA metabolism. NF90 was found to stabilize several mRNAs, including those that encode p21/CDKN1A, interleukin 2 (IL2), vascular endothelial growth factor (VEGF), and mitogen-activated protein kinase phosphatase 1 (MKP-1/DUSP1), typically by binding their $3^{\prime}$ UTRs (22). NF90 also repressed the translation of several mRNAs, including those that encode cytokines and chemokines essential for inflammation and senescence-associated secretory phenotype (SASP) observed in many primary cells that have ceased division in response to telomere attrition or have encountered other sublethal damage. In proliferating cells, high levels of NF90 helped suppress the translation of SASP factors MCP1/CCL2, GROa/CXCL1, IL6 and IL8, while in senescent cells, which expressed very low levels of NF90, translation of the same SASP factors was strongly elevated $(13,15)$. In the context of senescence, SASP and proinflammatory cytokines, it is interesting to note that NF90 suppressed the processing of several primary microRNAs, including pri-let-7, thereby reducing the levels of the potent growthinhibitory microRNA let-7 $(23,24)$ and potentially delaying the onset of a senescent phenotype.

Given that BAFF is a vital cytokine for B cells, the regulatory paradigm described here strengthens the involvement of NF90 in controlling immunity and the production of cytokines $(13,15,22)$. In this regard, NF90 was proposed to have a direct role in regulating Psoriasis vulgaris (25), an autoimmune disease influenced by abnormal $\mathrm{B}$ cell function (26). In sum, NF90 influences the immune response by controlling the production of immune factors including BAFF.

The finding that NF90 functions jointly with miR-15a to suppress BAFF translation critically advances our understanding of the molecular mechanisms underlying our earlier results that the BAFF-var genotype elevated increased soluble BAFF, increasing the risk of autoimmunity (8). The translation of $B A F F-W T$ mRNA (bearing two miR$15 \mathrm{a}$ sites, one on each side of the APA) was strongly suppressed by miR-15a, while BAFF-var mRNA translation (bearing only one miR-15a site located $5^{\prime}$ of the APA) was less affected. BAFF-var mRNA lacks one miR-15a binding site and therefore it is subject to less repression and produces more BAFF protein constitutively. Since only $B A F F$ $W T$ mRNA can jointly harbor both NF90 and two miR$15 \mathrm{a}$, only $B A F F-W T$ mRNA would be expected to be subject to robust cooperative repression. By contrast, $B A F F$ var mRNA would be refractory to repression and would be more actively translated, giving rise to high levels of secreted BAFF as measured in SardiNIA donors with the BAFF-var genotype, who show high propensity to autoimmune disorders such as MS and SLE, linked to elevated BAFF concentrations (8). Our results also point to new therapeutic strategies which might be devised to strengthen NF90 binding to $B A F F \mathrm{mRNA}$ to repress BAFF translation, raise miR-15a levels, or both, as we seek to intervene therapeutically to reduce the levels of factors that trigger or sustain autoimmunity states.

\section{SUPPLEMENTARY DATA}

Supplementary Data are available at NAR Online.

\section{FUNDING}

NIA-IRP, NIH; [N01-AG-1-2109 and HHSN271201 100005C]; Italian Foundation for Multiple Sclerosis [FISM 2011/R/13 and 2015/R/09]; Fondazione di Sardegna (ex Fondazione Banco di Sardegna, Prot. U1301.2015/AI.1157.BE Prat. 2015-1651). The open access publication charge for this paper has been waived by Oxford University Press - NAR Editorial Board members are entitled to one free paper per year in recognition of their work on behalf of the journal.

Conflict of interest statement. None declared.

\section{REFERENCES}

1. Orphanides,G. and Reinberg,D. (2002) A unified theory of gene expression. Cell, 108, 439-451.

2. Glisovic,T., Bachorik,J.L., Yong,J. and Dreyfuss, G. (2008) RNA-binding proteins and post-transcriptional gene regulation. FEBS Lett., 582, 1977-1986.

3. Bartel,D.P. (2009) MicroRNAs: target recognition and regulatory functions. Cell, 136, 215-233.

4. Krol,J., Loedige,I. and Filipowicz,W. (2010) The widespread regulation of microRNA biogenesis, function and decay. Nat. Rev. Genet., 11, 597-610.

5. Ivanov,P. and Anderson,P. (2013) Post-transcriptional regulatory networks in immunity. Immunol. Rev., 253, 253-272.

6. Bisogno,L.S. and Keene,J.D. (2017) RNA regulons in cancer and inflammation. Curr. Opin. Genet. Dev., 48, 97-103.

7. Kochi,Y. (2016) Genetics of autoimmune diseases: perspectives from genome-wide association studies. Int. Immunol., 28, 155-161.

8. Steri,M., Orrù,V., Idda,M.L., Pitzalis,M., Pala,M., Zara,I., Sidore,C., Faà,V., Floris,M., Deiana,M. et al. (2017) Overexpression of the Cytokine BAFF and autoimmunity risk. N. Engl. J. Med., 376, $1615-1626$.

9. Schneider,P. and Tschopp,J. (2003) BAFF and the regulation of B cell survival. Immunol. Lett., 88, 57-62.

10. Gross,J.A., Johnston,J., Mudri,S., Enselman,R., Dillon,S.R., Madden,K., Xu,W., Parrish-Novak,J., Foster,D., Lofton-Day,C. et al. (2000) TACI and BCMA are receptors for a TNF homologue implicated in B-cell autoimmune disease. Nature, 404, 995-999.

11. Mackay,F. and Schneider,P. (2009) Cracking the BAFF code. Nat. Rev. Immunol., 9, 491-502.

12. Rauch,M., Tussiwand,R., Bosco,N. and Rolink,A.G. (2009) Crucial role for BAFF-BAFF-R signaling in the survival and maintenance of mature B cells. PLoS One, 4, e5456.

13. Kuwano,Y., Pullmann,R. Jr, Marasa,B.S., Abdelmohsen,K., Lee,E.K., Yang,X., Martindale,J.L., Zhan,M. and Gorospe,M. (2010) NF90 selectively represses the translation of target mRNAs bearing an AU-rich signature motif. Nucleic Acids Res., 38, 225-238.

14. Panda,A.C., Martindale,J.L. and Gorospe,M. (2017) Polysome fractionation to analyze mRNA distribution profiles. Biol. Protoc. 7. e2126.

15. Tominaga-Yamanaka,K., Abdelmohsen,K., Martindale,J.L., Yang,X., Taub,D.D. and Gorospe,M. (2012) NF90 coordinately 
represses the senescence-associated secretory phenotype. Aging, 4, 695-708.

16. Tanguay,R.L. and Gallie,D.R. (1996) Translational efficiency is regulated by the length of the $3^{\prime}$ untranslated region. Mol. Cell. Biol., 16, $146-156$

17. Doran,G. (2008) The short and the long of UTRs. J. RNAi Gene Silencing, 4, 264-265.

18. Ciafrè,S.A. and Galardi,S. (2013) microRNAs and RNA-binding proteins: a complex network of interactions and reciprocal regulations in cancer. RNA Biol., 10, 935-942.

19. Ho,J.J. and Marsden,P.A. (2014) Competition and collaboration between RNA-binding proteins and microRNAs. Wiley Interdiscip. Rev. RNA., 5, 69-86.

20. Kedde,M., van Kouwenhove,M., Zwart,W., Oude Vrielink,J.A. Elkon,R. and Agami,R. (2010) A Pumilio-induced RNA structure switch in p27-3' UTR controls miR-221 and miR-222 accessibility. Nat. Cell Biol., 12, 1014-1020.

21. Yoon,J.H., Abdelmohsen,K., Srikantan,S., Yang,X., Martindale,J.L., De,S., Huarte,M., Zhan,M., Becker,K.G. and Gorospe,M. (2012)
LincRNA-p21 suppresses target mRNA translation. Mol. Cell, 47, 648-655.

22. Castella,S., Bernard,R., Corno,M., Fradin,A. and Larcher,J.C. (2015) Ilf3 and NF90 functions in RNA biology. Wiley Interdiscip. Rev. $R N A, \mathbf{6}, 243-256$.

23. Sakamoto,S., Aoki,K., Higuchi,T., Todaka,H., Morisawa,K., Tamaki,N., Hatano,E., Fukushima,A., Taniguchi,T. and Agata,Y. (2009) The NF90-NF45 complex functions as a negative regulator in the microRNA processing pathway. Mol. Cell. Biol., 29, 3754-3769.

24. Masuda,K. (2013) NF90 in posttranscriptional gene regulation and MicroRNA biogenesis. Int. J. Mol. Sci., 14, 17111-17121.

25. Stuart,P.E. (2015) Genome-wide association analysis of psoriatic arthritis and cutaneous psoriasis reveals differences in their genetic architecture. Am. J. Hum. Genet., 97, 816-836.

26. Hayashi,K. (2016) Gemcitabine enhances rituximab-mediated complement-dependent cytotoxicity to B cell lymphoma by CD20 upregulation. Cancer Sci., 107, 682-668. 